This item was submitted to Loughborough's Research Repository by the author.

Items in Figshare are protected by copyright, with all rights reserved, unless otherwise indicated.

\title{
A two-hop amplify-and-forward scheme for extended orthogonal space time coding in cooperative networks
}

PLEASE CITE THE PUBLISHED VERSION

PUBLISHER

(c) IEEE

VERSION

VoR (Version of Record)

\section{LICENCE}

CC BY-NC-ND 4.0

\section{REPOSITORY RECORD}

Eltayeb, Nasr E.M., Shakiru K. Kassim, and Jonathon Chambers. 2019. "A Two-hop Amplify-and-forward Scheme for Extended Orthogonal Space Time Coding in Cooperative Networks". figshare. https://hdl.handle.net/2134/5382. 
This item was submitted to Loughborough's Institutional Repository (https://dspace.lboro.ac.uk/) by the author and is made available under the following Creative Commons Licence conditions.

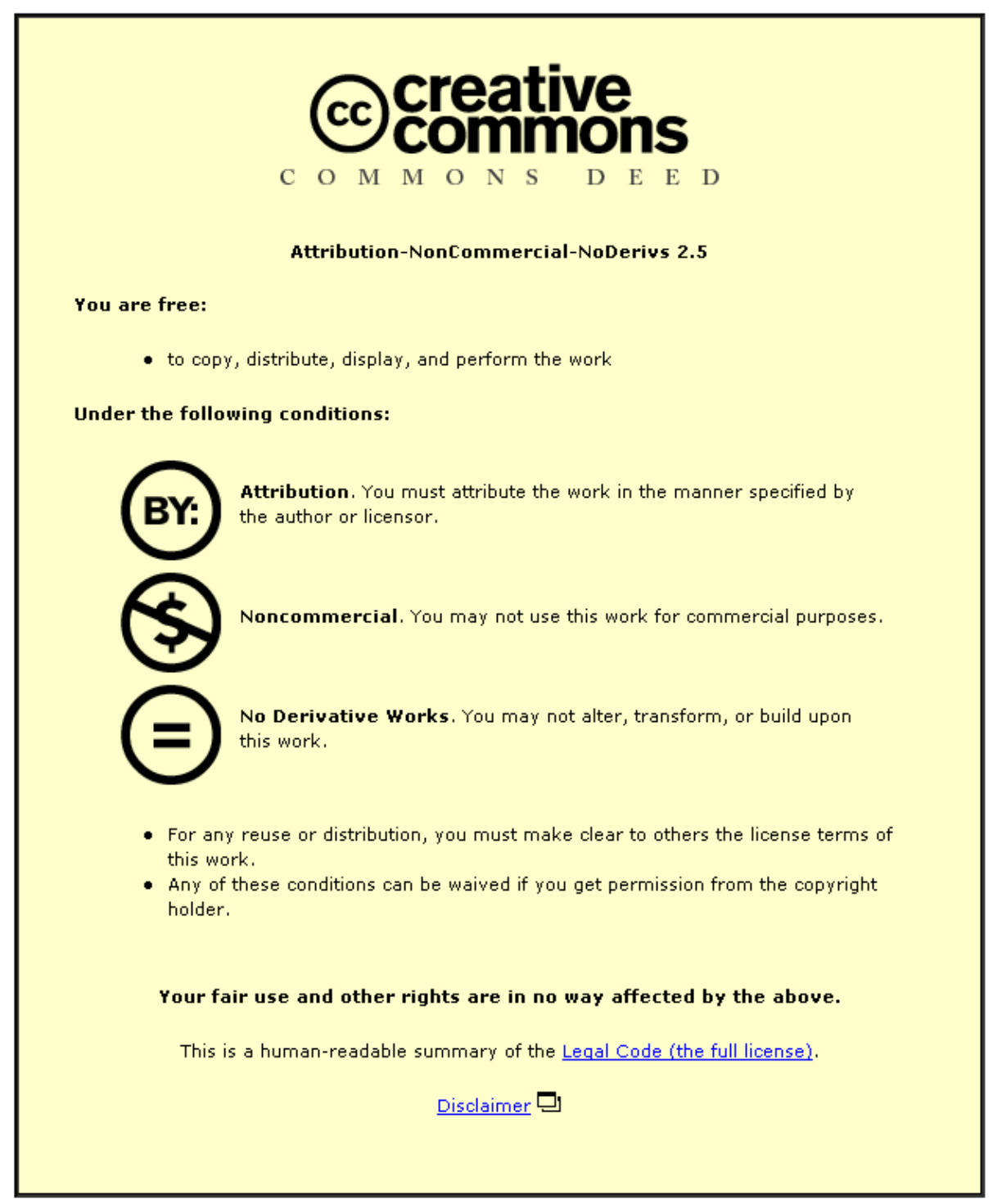

For the full text of this licence, please go to: http://creativecommons.org/licenses/by-nc-nd/2.5/ 


\title{
A Two-Hop Amplify-and-Forward Scheme for Extended Orthogonal Space Time Coding in Cooperative Networks
}

\author{
Nasr ELTAYEB, Shakiru KASSIM and Jonathon CHAMBERS
}

\author{
Faculty of Engineering \\ Dept. of Electronic and Electrical Engineering \\ Loughborough University, LE11 3TU, United Kingdom \\ E-mail: \{n.e.eltayeb, s.k.kassim, j.a.chambers\}@lboro.ac.uk
}

\begin{abstract}
We investigate and propose a new amplify-andforward cooperative relay networks scheme in which a full-rate and full-diversity extendedorthogonal space-time block coding (EOSTBC) scheme is used. Utilizing a feedback channel, a simple phase rotation is applied at the relay nodes to extract full diversity and array gain. A feedback quantization approach which reduces the overhead in the feedback channel for practical systems is also proposed. The performance of the proposed scheme, with and without optimum power allocation strategy, is investigated. Average bit error rate simulations confirm the utility of the scheme. The results also confirm the improvement in bit error rate (BER) performance over quasi-orthogonal space time block codes (QOSTBC).
\end{abstract}

\section{INTRODUCTION}

Cooperative communication has recently gained a lot of interest due to its ability to realize the performance gains of multiple-input multiple-output (MIMO) wireless systems, with distributed single antenna terminals forming virtual antenna arrays. More importantly, with the increasing interests in ad hoc networks, researchers have been looking for methods to exploit spatial diversity using the available "free" mobile terminals working as relays (because the fading paths from two mobiles are statistically independent, this generates spatial diversity), as well as splitting the available time slot into two phases [1][4]. The source node transmits during the first phase, while relays transmit during the second phase, sending a version of the received signal to the intended destination, thereby increase diversity order against fading and interference. Because original signals need two hops before arriving at destination in these system models, we can name them dual-hop cooperative systems.

In this paper, we consider a relay network with twostep protocol and apply EO space-time code [7] among the relays. The problem we are interested in is: "Can we increase the reliability of a wireless network by using EO space-time codes among the relays?" More specifically, the focus of this paper is on end-to-end BER analysis of wireless relay networks. We investigate in the diversity gain and array gain that can be achieved in wireless relay network by having parallel relays cooperate distributively. Here, by diversity gain we mean the negative of the exponent of the SNR or transmit power in the BER formula at hight SNR wich is consistent with the diversity gain definition in MIMO systems

On the assumption that relays are in full cooperation and adequately spaced to avoid interference, we demonstrated that with and without signal power control between the relaying stages, the proposed closed-loop EOSTBC (CL-EOSTBC) scheme outperforms closed-loop QOSTBC (CL-QOSTBC) scheme.

The organization of this paper is as follows. The system model is described in Section 2. In Section 3 we provide the power allocation strategy for the two-stage relay network. The results are verified by simulations in Section 4 using both QOSTBC and EOSTBC based on phase rotation feedback methods. In Section 5 we give the conclusions.

\section{SYSTEM MODEL}

In our model Fig.1, we consider a simple two-stage relay network, with a single source and destination communicating via cooperating relay nodes in parallel. All participating nodes communicate using a single antenna configuration over a quasi-static flat fading 


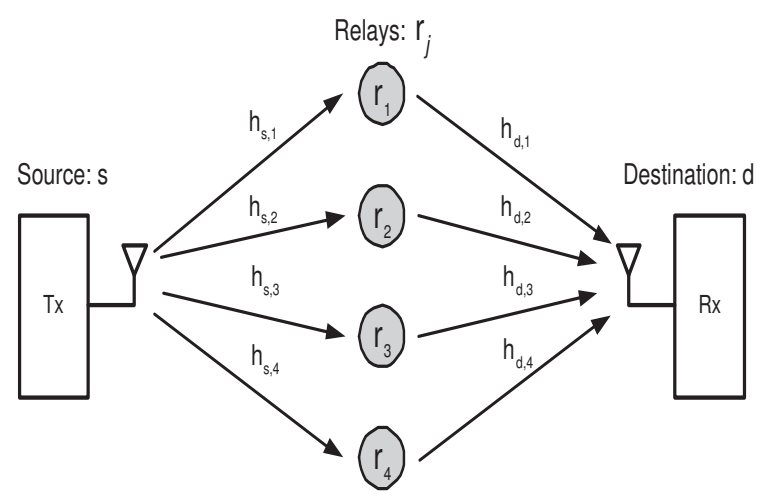

Figure 1: A simple two-hop cooperative relay network architecture:Amplify-and-Forward scheme.

channels $h_{i, j}$, where $i \in\{s, d\}$ represents the channel from the source or destination to a particular relay node $j \in\{1,2, \cdots, N\}$ up to the maximum number of participating relays $\mathrm{N}$. In the context of this work all random channel parameters $h_{i, j}$ are assumed to be zero mean circular complex Gaussian (ZMCSCG) random variable with unity variance (i.e Rayleigh fading). In this scheme we use a simple two-step protocol over two time frames, i.e time division multiple access (TDMA). In the first time frame the source node broadcasts $\sqrt{P_{s}} s_{k}$ to the relay nodes over a number of symbol intervals depending on the size of the STBC coding matrix; where $P_{s}$ is the source transmit power and $s \in Z$ is the transmitted symbol. Assuming perfect cooperation among relay nodes, i.e. perfect synchronization the distributed channel vector $\mathbf{h}_{s r}$ can take the form,

$$
\mathbf{h}_{s, i}=\left[h_{s, 1} h_{s, 2} \cdots h_{s, N}\right]^{T}
$$

At the relay nodes, the received signals at the $i t h$ relay is denoted as $y_{i}$, which is corrupted by both the fading $h_{s, i}$ and the noise $n_{s, i}$.

$$
\mathbf{y}_{i}=\sqrt{P_{s}} \mathbf{h}_{s, i} s+\mathbf{n}_{\mathbf{s}, \mathbf{i}}
$$

In the next time-frame each relay node synchronously re-transmits a scaled version of the received signals in the first time-frame with the same power constraint as in the first hop, according to a pre-allocated column of a STBC matrix, whereas the source remains silent. In this implementation the EOSTBC scheme [6] with four participating relays is used,

$$
\mathcal{G}=\gamma_{i}\left[\begin{array}{cccc}
y_{1} & y_{1} & y_{2} & y_{2} \\
-y_{2}^{*} & -y_{2}^{*} & y_{1}^{*} & y_{1}^{*}
\end{array}\right]
$$

where $\gamma_{i}$, denote the average transmission power at every relay nodes and (.)* denotes complex conjugate operation. However in [2], Akhter et al. note that full order diversity cannot be accomplished due to interference factor between estimated symbols, illustrated by the $\hat{\beta}$ terms below, which after combining at the destination receiver results in a matrix of the following form,

$$
\mathbf{H}_{r d}^{H} \mathbf{H}_{r d}=\left[\begin{array}{cc}
\hat{\alpha}+\hat{\beta} & 0 \\
0 & \hat{\alpha}+\hat{\beta}
\end{array}\right]
$$

For a more detailed analysis the interested reader is referred to [2] and [3]. However, Eltayeb et al. [7] showed that a simple phase rotation $\theta$ and $\phi$ on the complex symbols transmitted from nodes 1 and 3 ensure that not only the $\hat{\beta}$ term is positive but also maximized during the transmission period, which will provide $4 t h$ order diversity and array gain, at the expense of requiring a feedback channel.

Following similar approach, a coding matrix was designed for the QOSTBC [8] as:

$$
\mathcal{Q}=\gamma_{i}\left[\begin{array}{cccc}
y_{1} & y_{2} & y_{3} & y_{4} \\
-y_{2}^{*} & y_{1}^{*} & -y_{4}^{*} & y_{3}^{*} \\
-y_{3}^{*} & -y_{4}^{*} & y_{1}^{*} & y_{2}^{*} \\
y_{4} & -y_{3} & -y_{2} & y_{1}
\end{array}\right]
$$

where $\gamma_{i}$ is the transmit power in each relay. Both schemes explore a phase feedback from the destination node to the relays in order to exploit the full diversity, hence CL-EOSTBC and CL-QOSTBC.

The received signal $r_{d}$ at the destination can be described as

$$
r_{d}=\sum_{i=1}^{N=4} h_{d, i} y_{i}+n_{d, i}
$$

where $h_{d, i}$ is the channel gain between the relay $r_{i}$ and destination $d, y_{i}$ is the encoded received signal plus noise at relay $r_{i}$ of $s_{i}$ and $n_{d, i}$ is the complex Gaussian noise with mean zero and variance $N_{0} / 2$ per-dimension.

\section{Resource-Allocation Strategy}

In this section, we discuss the optimum power allocated among the source and the relays to maximize the received SNR at the destination.

Under the assumption of full data exchange, each of the $K$ relaying stages experiences independent BERs, which are denoted here as $P_{b, v \in(1, K)}(e)$, caused by independent SERs $P_{s, v \in(1, K)(e)}$. A bit from the source terminal is received correctly at the target only when at all stages, the bit has been transmitted correctly.

The end-to-end BER can therefore be expressed as,

$$
P_{b, e 2 e}(e)=1-\prod_{v=1}^{K}\left(1-P_{b, v}(e)\right)
$$


which at low BER or sufficiently high SNRs at every stage, can be written as,

$$
\begin{aligned}
P_{b, e 2 e}(e) & =\sum_{v=1}^{K} P_{b, v}(e) \\
& =\sum_{v=1}^{K} \frac{P_{s, v}(e)}{\log _{2}\left(M_{v}\right)}
\end{aligned}
$$

where $M_{v}$ is the modulation order at the $v t h$ stage.

To maximize the capacity and hence to minimize the end-to-end BER we allocate optimal power to each stage, the total transmission power available in the system satisfy the following power constraint,

$$
\beta_{1}+\sum_{i=1}^{n=4} \beta_{i}=P
$$

where $\alpha_{1} P$ and $\alpha_{i} P, i=1, \cdots, 4$ are the power allocated to the source and the ith relay, respectively, and their sum should not exceed the overall network power constraint $P$ as shown in (9).

If the source transmits $B$ bits per frame, where the frame is defined as $D=B / \log _{2}(M)$, to the target over $k$ relaying stages, the normalized end-to-end throughput $\Theta($ Bits $/ \mathrm{Hz})$ can be expressed as,

$$
\Theta=\min _{v \in(1, K)}\left\{\alpha_{v} R_{v} \log _{2}\left(M_{v}\right)\right\} \cdot\left(1-P_{b, e 2 e}(e)\right)^{B}
$$

where $R_{v}$ is the STBC rate of the $v t h$ stage, $M_{v}$ is the modulating index, $\alpha_{v}$ is the optimized frame duration of the $v$ th stage under the constraint that $\sum_{v=1}^{k} \alpha_{v}=1$, $P_{b, e 2 e}(e)$ is the end-to-end BER and $\min \{$.$\} signifies$ the dependence of the throughput on the weakest link in the chain.

The end-to-end throughput is maximized by minimizing the end-to-end BER through optimally assigning fractional power allocated to each relaying stage with our assumption that the fractional frame duration is optimized.

The elegant analysis presented in [4] simplifies BER by invoking its upper bound which occurs when (8) is upper bounded by its largest argument $\theta=\pi / 2$.

$$
P(e)=\frac{1}{\pi} \int_{0}^{\pi \frac{M-1}{M}} \phi_{\rho}\left(\frac{G P S K}{\sin ^{2} \theta}\right) d \theta
$$

where $M$ is the constellation size and GPSK $\equiv$ $\sin ^{2}(\pi / M)$ and thus the presented BER of the $v t h$ relaying stage for $M$-PSK ${ }^{1}$ can be upper bounded as,

\footnotetext{
${ }^{1}$ The result can be applied to other binary and M-ary signals in a straightforward way (see e.g. [4]).
}

$$
\begin{gathered}
P_{b, e 2 e}(e) \leq \sum_{v=1}^{K} \frac{M_{v}-1}{M_{v} \log _{2}\left(M_{v}\right)}\left(1+\beta_{v} \frac{G P S K}{R_{v}} \frac{\gamma_{v}}{t_{v}} \frac{S}{N}\right)^{-q} \\
P_{b, e 2 e}(e) \leq \sum_{v=1}^{K} A_{v}\left(1+B_{v} \beta_{v}\right)^{-q}
\end{gathered}
$$

This upper bound was then utilized to determine the effective fractional allocated power $\beta_{v \in(1, K)}$ that obeys

$$
\beta_{v}=\left[\alpha_{w}\left(\frac{q_{v}^{-1} A_{v}^{-1} B_{v}^{q_{v}}}{q_{w}^{-1} A_{w}^{-1} B_{w}^{q_{w}}}\right)^{\frac{1}{q_{a x}+1}}\right]^{-1}
$$

where $q_{\max }=\arg \max \left(q_{1}, \cdots, q_{k}\right)$ and $w$ is the previous relaying stage.

The constant $A_{v}$ and $B_{v}$ are obtained by comparing (13) with (12) to arrive at

$$
\begin{aligned}
& A_{v}=\frac{M_{v}-1}{M_{v} \log _{2}\left(M_{v}\right)} \\
& B_{v}=\frac{G P S K_{v}}{R_{v}} \frac{\gamma_{v}}{t_{v}} \frac{S}{N}
\end{aligned}
$$

where the fractional frame durations $\alpha_{w}$ need to be chosen such as to maximize (10) under the constraint $\sum_{v=1}^{K} \alpha_{v}=1$. This is clearly achieved by

$$
\alpha_{w}=\frac{\prod_{w=1, w \neq v}^{K} R_{w} \cdot \log _{2}\left(M_{w}\right)}{\sum_{k=1}^{K} \prod_{w=1, w \neq k}^{K} R_{w} \cdot \log _{2}\left(M_{w}\right)}
$$

The performance of the algorithm is assessed by means of simulation for M-PSK schemes only. We explicitly show the end-to-end BER versus the SNR for various 2-stage communication scenario deploying the demonstrated fractional power allocation strategy in (14), which is also compared against a non-optimum power allocation scheme.

\section{SIMULATION RESULTS}

In this section we provide simulation results for our proposed scheme. We consider a QPSK modulation in our simulations. For all our simulation results we assume perfect CSI and equal signal-to-noise ratio (SNR) at all receiving terminal, i.e. a terminal knows perfectly the channel state information of the link preceding it. We assume that the channel is quasi-static flat fading time-invariant during the transmission of one symbol period. We also assume that the channel is quasi-static flat fading time-invariant during the transmission of one symbol period. 


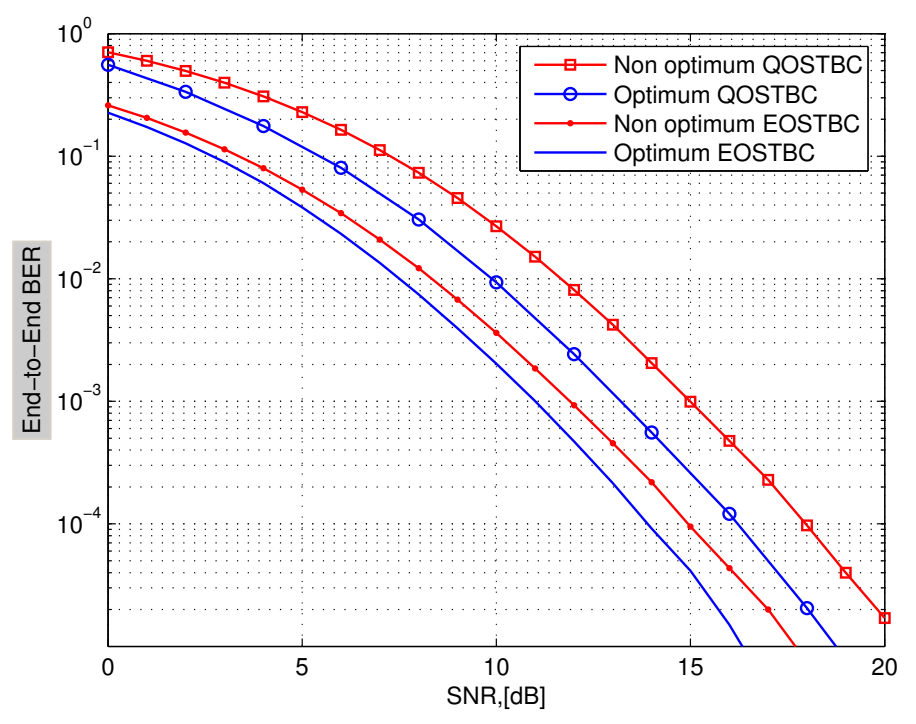

Figure 2: Comparison between optimum and non-optimised end-to-end BER for various configurations of a two-stage relaying networks over frequency-flat fading channels.

A simple relay network based on quasi-orthogonal STBC (QOSTBC) scheme using an AAF protocol at the relays was proposed in [9]. Figure 2 compares our proposed relay network based on extended-orthogonal STBC (EOSTBC) scheme for both optimum and nonoptimum scenarios with an optimum and non-optimum QOSTBC implementation and illustrates the performance improvements achieved by phase rotation at the relay nodes, i.e nodes 1 and 3 . From the figure we can see that the performance of the proposed system is always better than the that of the relay network based QOSTBC at any power or SNR. Also we can see from figure 2 that the difference of the slope of the nonoptimum and optimum curves of the two systems are increasing as the total transmit power goes higher.

A further important issue for real-world applications is what happens to the over all performance of the optimum relay network based EOSTBC scheme if the power control routine fails to operate properly. There will be only $0.5 \mathrm{~dB}$ loss in the performance of the proposed scheme which still significantly performs better than the QOSTBC relaying link with optimum power allocation; therefore ensuring a more robust transmission scheme in the event of power control failure.

Finally, the simulation result of a practical scenario of a quantized method is depicted in Figure 3. We notice that the performance gain of the quantized scheme and the ideal feedback scheme are perfectly match-

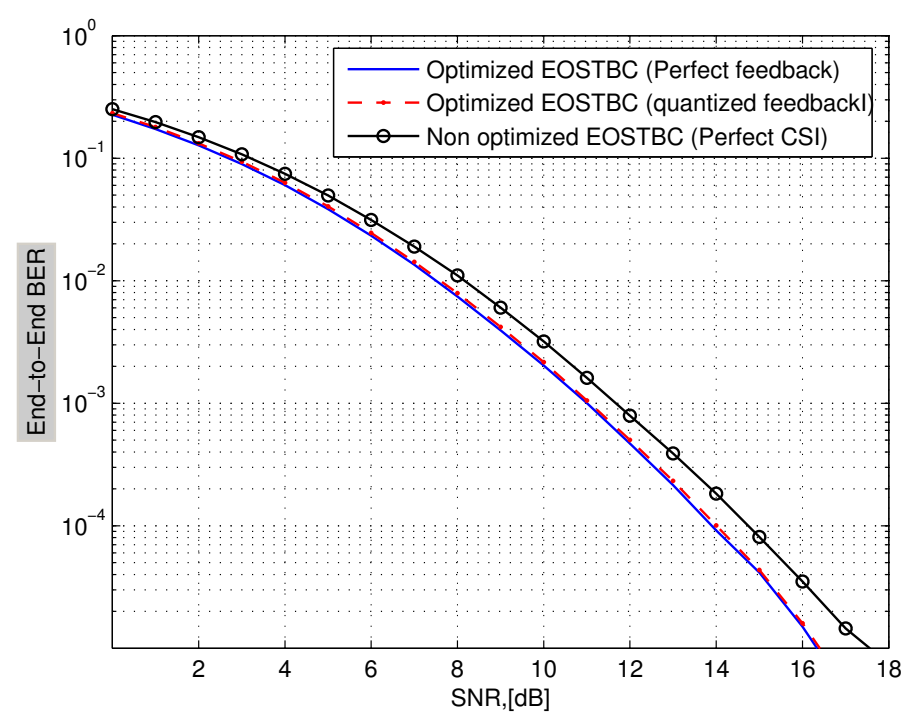

Figure 3: Optimum end-to-end BER of the proposed scheme of two-stage relaying network with feedback quantization approach.

ing and still considerably better than the non-optimum case.

\section{COCLUSION}

In this paper, we study the optimal power allocation among the source and the relays in a wireless relay network. We consider AAF relay network for both QOSTBC and EOSTBC. The optimal power allocation for these schemes were demonstrated. Simulation results show that the optimum power allocation can bring the proposed scheme a considerable received SNR gains compared to equal power allocation. This implies that the optimum power allocation scheme is very effective in optimizing the system performance and reducing the overall consuming power of a wireless network.

\section{References}

[1] A. Sendonaris, E. Erkip, and B. Aazhang: "User cooperation diversity-part I: System description," IEEE Trans. Commun., vol.51, no.11, pp.1927-1938, Nov. 2003.

[2] J. Akhtar and D. Gesbert; "Extended Orthogonal Block Codes With Partial Feedback," IEEE Transactions on Wireless Communications, vol.3, no.6, , pp.1959-1962, Nov. 2004. 
[3] Y. Yu S. Kerouden, and J. Yuan; "Closed-Loop Extended Orthogonal Space-Time Block Codes for Three and Four Tranmsit antennas," IEEE Signal Processing Letters, vol.13, no.5, pp.273276, May. 2006.

[4] M. Dohler: "Virtual Antenna Array" PhD Thesis, University of London, Nov. 2003.

[5] Y. Jing and B. Hassibi: "Distributed space-time coding in wireless relay networks" IEEE Transactions on Communications, vol.5, no.12, Dec. 2006.

[6] J. N. Laneman, D. N. C. Tse, and Gr. W.Wornell: "Cooperative diversity in wireless networks: Efficient protocols and outage behavior," IEEE Trans. Inform. Theory, vol.50, no.12, pp.30623080, Dec. 2004.

[7] N. M. Eltayeb, S. Lambotharan, and J. A. Chambers: "A Phase Feedback Based Extended SpaceTime Block Code for Enhancement of Diversity," IEEE VTC Conference, 65th, pp.22962299, April 2007.

[8] H. Jafarkhani: "A Quasi-Orthogonal Space-Time Block Code", IEEE Trans. Commun., vol.49, pp.1-4, Jan. 2001.

[9] S.K.Kassim, M.Hayes, N.M. Eltayeb, J.A. Chambers, "Exploitation of Quasi-Orthogonal Space Time Block Codes in Virtual Antenna Arrays: Part II Monte Carlo-Based throughput Evaluation," IEEE Vehicular Technology Conference, 66th, pp.242-246, May 2008. 